\title{
The cosmological dark sector as a scalar $\sigma$-meson field
}

\author{
Saulo Carneiro ${ }^{1,2, \mathrm{a}_{\mathbb{C}}}$ \\ ${ }^{1}$ Instituto de Física Gleb Wataghin, UNICAMP, 13083-970 Campinas, SP, Brazil \\ 2 Instituto de Física, Universidade Federal da Bahia, 40210-340 Salvador, BA, Brazil
}

Received: 18 December 2017 / Accepted: 22 February 2018 / Published online: 5 March 2018

(C) The Author(s) 2018. This article is an open access publication

\begin{abstract}
Previous quantum field estimations of the QCD vacuum in the expanding space-time lead to a dark energy component scaling linearly with the Hubble parameter, which gives the correct figure for the observed cosmological term. Here we show that this behaviour also appears at the classical level, as a result of the chiral symmetry breaking in a low energy, effective $\sigma$-model. The dark sector is described in a unified way by the $\sigma$ condensate and its fluctuations, giving rise to a decaying dark energy and a homogeneous creation of non-relativistic dark particles. The creation rate and the future asymptotic de Sitter horizon are both determined by the $\sigma$ mass scale.
\end{abstract}

The cosmological constant problem is often stated as a large, non-observed contribution of the vacuum of quantum fields to the dark energy component of the cosmic fluid. Although very popular, this statement turns the problem ill defined, because the quantum field calculations are usually performed in the flat space-time, where nothing would really gravitate. A more meaningful procedure is to obtain the vacuum contribution in the curved, FLRW space-time, with a reliable renormalisation that absorbs the divergent flat result in the constants of the theory [1-5]. This can be made, for example, in the case of conformal fields, which naturally leads to a vacuum density of the order of $H^{4}$ ( $H$ is the Hubble function), since no other scale is available. This result is useful, in the high energy limit, to obtain non-singular cosmological solutions [6,7]. In the present universe, however, its figure has the opposite problem of being too small as compared to the observed cosmological term. More generally, other even powers of $H$ can result from these calculations, owing to a theorem on the allowed counter terms in the effective action [8]. For instance, a free field of mass $m$ may lead to a contribution of order $m^{2} H^{2}$, which has the observed figure if $m$ has the order of the Planck mass. However, with a dark energy scaling with $H^{2}$, the cosmic expansion would not experience the observed transition from decelerating to

a e-mail: saulo.carneiro@pq.cnpq.br accelerating phases. The remaining possibility is a constant $\Lambda$, which value, however, is not fixed by the theory.

The theorem mentioned above has, as always, its premises. Among them we can find the conditions that the fields are free, and that the vacuum energy-momentum tensor is conserved. The first does not apply, for example, if we estimate the vacuum contribution of the low energy, strongly coupled fields of QCD. Indeed, some studies have suggested that these fields lead to a vacuum term scaling as $m_{\pi}^{3} H$, where $m_{\pi}$ is the energy scale of the chiral phase transition [9-15]. Surprisingly enough, this gives the observed order of magnitude for $\Lambda$. The second premise, as well, cannot be always satisfied. A decaying $\Lambda$ is only possible either if its equation of state parameter $\omega \neq-1$, or if it interacts with dark matter. As shown elsewhere [16-19], $\omega=-1$ is a necessary condition for $\Lambda$ to be strictly homogeneous, which permits an unambiguous definition of clustering dark matter. Therefore, a vacuum term decaying with $H$ is necessarily concomitant with a homogeneous creation of dark particles. The freedom in defining $\Lambda$ such that $\omega=-1$ is a manifestation of the so called dark degeneracy [16,20-22]. As we will see, this freedom allows a unified description of the dark sector in terms of a non-adiabatic scalar field, which plays the role of both dark energy and dark matter. In particular, we will show that the $\sigma$ scalar, that emerges from effective models of low energy QCD, produces indeed a dark energy scaling linearly with $H$.

In its simplest version, the $\sigma$ potential is given by [23]

$V=-\frac{\mu^{2}}{2} \sigma^{2}+\frac{\lambda}{8} \sigma^{4}+\tilde{V}$,

where $\mu, \lambda$ and $\tilde{V}$ are positive constants. It presents a false vacuum at $\sigma=0$ and a true vacuum at $\sigma=f$, with $f$ given by $\lambda f^{2}=2 \mu^{2}$. There is a priori no clear prescription for the choice of vacuum in cosmological backgrounds. Nevertheless, as our universe is approaching a de Sitter space-time with very small curvature, we assume that the flat space-time 
vacuum structure is not altered, except for the minimum of the potential at the true vacuum state, as we will show below. When the chiral symmetry is spontaneously broken, expanding the potential (1) around the vacuum expectation value $f$ leads, up to second order, to

$V(\phi)=V_{d S}+\frac{M^{2} \phi^{2}}{2}$,

where $\phi=\sigma-f$ are scalar fluctuations of the chiral condensate, with mass $M=\sqrt{2} \mu$, and

$V_{d S}=\tilde{V}-\frac{\mu^{2} f^{2}}{4}$

We will see that, in an FLRW background, $V_{d S}$ is also determined by $\mu$. The energy density and pressure of the scalar field are

$\rho_{\phi}=\frac{\dot{\phi}^{2}}{2}+V$,

$p_{\phi}=\frac{\dot{\phi}^{2}}{2}-V$,

where a dot means derivative w.r.t. the cosmological time. We can always decompose this perfect fluid into pressureless matter and a $\Lambda$ component [16,20-22], such that

$\rho_{m}=\dot{\phi}^{2}$

$\Lambda=-p_{\Lambda}=V-\frac{\dot{\phi}^{2}}{2}$.

In the general case, these two components interact and, in this way, the content above can be seen as a particular case of a non-adiabatic dark sector [24-41]. This decomposition assures that the $\Lambda$ component does not cluster, provided the matter component follows geodesics, i.e. provided there is no momentum transfer between the components [16-19]. We are going to show that this is indeed the case. Furthermore, as $\delta \Lambda=0$, the scalar-field effective sound speed $\delta p_{\phi} / \delta \rho_{\phi}$ is zero, which prevents, at the perturbation level, oscillations and instabilities in the matter power spectrum [16].

The energy-momentum balance between the two components can be written as

$T_{m ; v}^{\mu \nu}=Q^{\mu}$

$T_{\Lambda ; v}^{\mu \nu}=-Q^{\mu}$,

where

$Q^{\mu}=Q u^{\mu}+\bar{Q}^{\mu} \quad\left(\bar{Q}^{\mu} u_{\mu}=0\right)$

is the energy-momentum transfer, and $u^{\mu}$ is the fluid 4-velocity. The transverse component $\bar{Q}^{\mu}$ represents the momentum transfer. Writing for the $\Lambda$ term

$T_{\Lambda}^{\mu \nu}=\Lambda g^{\mu \nu}$, we have, from (9),

$Q=-\Lambda_{, v} u^{v}$,

$\bar{Q}^{\mu}=\Lambda_{, v}\left(u^{\mu} u^{v}-g^{\mu v}\right)$.

Perturbing these equations we obtain $\delta \bar{Q}_{0}=0$ and, in a co-moving gauge,

$\delta Q=-(\delta \Lambda), 0$,

$\delta \bar{Q}_{i}=(\delta \Lambda)_{, i}$.

Therefore, if we show that $\delta \bar{Q}^{\mu}$ is zero, we show that $\Lambda$ is strictly homogeneous (and, in addition, that $\delta Q=0$ ). Components (6) and (7) can be written, respectively, in the covariant form

$T_{m}^{\mu \nu}=\partial^{\mu} \phi \partial^{v} \phi$

$T_{\Lambda}^{\mu \nu}=\left(V-\frac{1}{2} \partial_{\alpha} \phi \partial^{\alpha} \phi\right) g^{\mu \nu}$.

From (17) and (9) we can write

$Q_{\mu}=\partial_{\alpha} \phi \partial^{\alpha} \partial_{\mu} \phi-V^{\prime} \partial_{\mu} \phi$

with $V^{\prime}=M^{2} \phi$ from (2). In Fourier's space it takes, in the adiabatic regime, ${ }^{1}$ the form

$Q_{\mu}=i k_{\mu}\left(M^{2}-k^{2}\right) \phi^{2}$,

where $k_{\mu}$ is the scalar-field wavevector. By doing $i k_{\mu}=k u_{\mu}$ we obtain

$Q_{\mu}=k\left(M^{2}-k^{2}\right) \phi^{2} u_{\mu}$,

which shows that $\bar{Q}^{\mu}=0$ in any space-time. ${ }^{2}$

The Friedmann equations for the spatially flat FLRW space-time are given by

$3 H^{2}=V+2 H^{\prime 2}$,

$\dot{\phi}=-2 H^{\prime}$,

where a prime means derivative w.r.t. the scalar field. On the other hand, by using (6) and (7), the Klein-Gordon equation,

$\ddot{\phi}+3 H \dot{\phi}+V^{\prime}=0$,

can be put in the form

$\dot{\rho}_{m}+3 H \rho_{m}=-\dot{\Lambda} \equiv \Gamma \rho_{m}$

\footnotetext{
${ }^{1}$ In the non-adiabatic regime $Q_{\mu}$ has additional terms due to the time dependence of $k_{0}$, but remains colinear to $u_{\mu}$.

2 Technically, any space-time admitting plane waves as a complete ortogonal basis, in particular the spatially flat FLRW space-time and its first order perturbations.
} 
The last equality defines the rate of matter creation $\Gamma$, which is a function to be determined. Using (6), (7), (22) and (23) into (24), we obtain

$V^{\prime}=(3 H+\Gamma) H^{\prime}$

With the potential given by (2), Eqs. (21) and (25) determine the solutions for $H(\phi)$ and $\Gamma(\phi)$. The second order solution for $H$ can be written as

$H=H_{d S}\left(1+A \phi^{2}\right)$,

where $A$ is a positive constant. It corresponds to a spacetime going to de Sitter as $\phi$ approaches the minimum of the potential. Using (2) and (26) into (25) we obtain, at the same order of approximation,

$\Gamma=\frac{M^{2}}{2 A H_{d S}}-3 H_{d S}$,

which means that matter is created at a constant rate. On the other hand, from (6), (22) and (24) we have

$\Lambda^{\prime}=2 \Gamma H^{\prime}$,

which, after integration, leads to

$\Lambda=2 \Gamma H+\tilde{\Lambda}$

where $\tilde{\Lambda}$ is an arbitrary integration constant. There is no energy scale fixing $\tilde{\Lambda}$, let us hence take it zero, that is,

$\Lambda=2 \Gamma H$

In the de Sitter limit $\phi \rightarrow 0$ we have, from (2), (21), (26) and (30),

$V_{d S}=3 H_{d S}^{2}=\frac{4 \Gamma^{2}}{3}$.

Substituting (26) into (21), retaining only terms up to second order in $\phi$ and using (31) we obtain

$\frac{32 \Gamma^{2}}{9} A^{2}-\frac{8 \Gamma^{2}}{3} A+\frac{M^{2}}{2}=0$.

On the other hand, using (31) into (27) we get

$M^{2}=4 \Gamma^{2} A$.

From (32) and (33) we then have $A=3 / 16$, which, substituted back into (33), gives

$\Gamma^{2}=\frac{4 M^{2}}{3}$
We see that $\sqrt{2} \mu=M$ completely determines not only the mass of $\phi$, but, through (31) and (34), also the matter creation rate $\Gamma$, the de Sitter horizon $H_{d S}$ and the potential (2).

The cosmological solution with constant-rate creation of matter was tested against observations at background and perturbation levels, showing a good approximation to the present universe [42-45]. ${ }^{3}$ For $\Gamma=0$ we have a Minkowski space-time with $V=0$, which is however unstable and eventually collapses to a singularity [48]. For $\Gamma \neq 0$ we have an expanding space-time, which tends asymptotically to a stable de Sitter universe. For high redshifts the matter density decreases with the scale factor as $a^{-3}$, as the matter creation rate is negligible compared to the expansion rate. Near the de Sitter phase it varies slowlier, as $a^{-3 / 2}$, due to matter creation [42-45]. This can also be seen from (16), which in Fourier's space gives $\rho_{m}=k^{2} \phi^{2}$. Near the de Sitter phase, the KleinGordon equation (23) has the solution $\phi \propto \exp (-k t)$, with $k=3 H_{d S} / 4$. Using the de Sitter solution $a \propto \exp \left(H_{d S} t\right)$ we obtain $\rho_{m} \propto a^{-3 / 2}$. On the other hand, from (20) it is easy to show, in the same limit, that $Q=\Gamma \rho_{m}$, which is consistent with the definition of $\Gamma$ given by (24).

As a matter of fact, the result (30) can be achieved with any free scalar field of mass $M$, since all we need is a potential like (2) and a space-time close enough to de Sitter [16]. Nevertheless, we may show that the QCD chiral phase transition leads naturally to the correct energy scale we need to describe the observed universe. From (30) we see that the constant $\Gamma$ has the dimension of the cube of a characteristic energy $m_{\pi}$. By taking (30) at the present time, we can write

$m_{\pi}^{3} \sim H_{0} \Omega_{\Lambda 0}$,

where $\Omega_{\Lambda 0} \sim 1$ is the dark energy density relative to the critical density. By taking also $H_{0} \sim 10^{-18} s^{-1}$, we have

$m_{\pi} \sim 100 \mathrm{MeV}$

which is the energy scale of the QCD phase transition. Leading $\Gamma \sim m_{\pi}^{3}$ into (34), we obtain the mass ${ }^{4}$

$M \sim 10^{-33} \mathrm{eV}$

On the other hand, if we assume that the proportionality between the mass of the chiral condensate fluctuations and the condensate density is also valid at nuclear scales [51], we can write

$\frac{M}{\rho_{c}} \sim \frac{m_{\sigma}}{\rho_{N u c}} \sim m_{\pi}^{-3}$,

3 The non-interacting version of the $\Lambda \propto H$ model is excluded by observations [46]. For non-interacting, scalar-field based decaying dark energy models, see, e.g., Ref. [47].

${ }^{4}$ The cosmological role of a pseudo Nambu-Goldstone boson with mass $H_{0}$ was already considered before, in other contexts $[49,50]$. 
where $\rho_{c}$ is the cosmological critical density, $m_{\sigma} \sim 1 \mathrm{GeV}$ is the observed $\sigma$ mass, and $\rho_{N u c}$ is a typical nuclear density. With $\rho_{c} \sim 10^{-26} \mathrm{~kg} / \mathrm{m}^{3}$ and $\rho_{N u c} \sim 10^{16} \mathrm{~kg} / \mathrm{m}^{3}$, we obtain for $M$ the same figure as in (37). It is also worthy of note that $M$ is the quantum of energy expected in the de Sitter space-time. Indeed, the number of degrees of freedom inside the de Sitter horizon is given by the holographic bound $N \sim$ $H_{d S}^{-2}$ [52-62]. The corresponding energy is $E \sim \rho H_{d S}^{-3} \sim$ $H_{d S}^{-1}$. Therefore, the energy per degree of freedom is $E / N \sim$ $H_{d S} \sim M$, by (31) and (34). It is also suggestive (see (31)) that the rate of dark particles creation $\Gamma$ has the order of the de Sitter horizon temperature $H_{d S}[63-65] .^{5}$

Models with dynamic dark energy, and with interactions in the dark sector, in particular, have been studied from both theoretical and observational viewpoints [17-19,24-47,66-70]. Some of them are essentially phenomenological [36-38,6670], while others present a field-based theoretical background (see, e.g., Refs. [39-41,47]). In the present contribution we have tried to link the dynamics of the dark sector to QCD vacuum estimations [9-15], on the basis of a low-energy, effective $\sigma$-model. It predicts, in the low-redshift limit, a dark energy density scaling linearly with $H$, phenomenology that has shown a good approximation to the present universe [4245]. Furthermore, allowing an interaction between the dark components has permitted to adopt a covariant equation of state for vacuum (see (11)), something not possible in the case of a conserved dynamical dark energy. This has also allowed an unambiguous definition of clustering matter, since vacuum does not cluster, as explicitly proved. In a subsequent work [71] we show that a scalar field can in fact describe not only the model with $\Lambda \propto H$, but a family of solutions that are equivalent to non-adiabatic generalised Chaplygin gases [36-38,66-70]. In spite of the theoretical motivations of the present model, only observations will determine whether any interaction actually exists, and its precise form.

Acknowledgements I am thankful to M. R. Robilotta, C. Chirenti, H. A. Borges, T. S. Pereira and W. Zimdahl for helpful discussions, to G. A. Mena Marugán, J. S. Alcaniz and J. C. Fabris for critical readings, and to CNPq for financial support (grant no. 309792/2014-2).

Open Access This article is distributed under the terms of the Creative Commons Attribution 4.0 International License (http://creativecomm ons.org/licenses/by/4.0/), which permits unrestricted use, distribution, and reproduction in any medium, provided you give appropriate credit to the original author(s) and the source, provide a link to the Creative Commons license, and indicate if changes were made. Funded by SCOAP ${ }^{3}$.

\footnotetext{
5 The term "dark particle" is an abuse of language. As we have seen, in the de Sitter limit the solution of the Klein-Gordon equation has imaginary frequency. The fluctuations $\phi$ have wavelengths of the order of the Hubble horizon and we cannot define a particle occupation number. As well as dark energy, dark matter should be better interpreted as a field in this context.
}

\section{References}

1. S.A. Fulling, L. Parker, Phys. Rev. D 10, 3905 (1974)

2. L.H. Ford, Phys. Rev. D 11, 3370 (1975)

3. J.S. Dowkler, R. Critchley, Phys. Rev. D 13, 3224 (1976)

4. P.C. Davies, Phys. Lett. B 68, 402 (1977)

5. A.A. Starobinsky, Phys. Lett. B 91, 99 (1980)

6. S. Carneiro, R. Tavakol, Gen. Relat. Gravit. 41, 2287 (2009)

7. L.P. Chimento, S. Carneiro, AIP Conf. Proc. 1647, 10 (2015)

8. R.M. Wald, Quantum Field Theory in Curved Spacetime and Black Hole Thermodynamics (The University of Chicago Press, Chicago, 1994). (Section 4.6)

9. R. Schutzhold, Phys. Rev. Lett. 89, 081302 (2002)

10. F.R. Klinkhamer, G.E. Volovik, Phys. Rev. D 79, 063527 (2009)

11. F.R. Urban, A.R. Zhitnitsky, Phys. Lett. B 688, 9 (2010)

12. F.R. Urban, A.R. Zhitnitsky, Nucl. Phys. B 835, 135 (2010)

13. N. Ohta, Phys. Lett. B 695, 41 (2011)

14. B. Holdom, Phys. Lett. B 697, 351 (2011)

15. R.G. Cai, Z.L. Tuo, H.B. Zhang, Q. Su, Phys. Rev. D 84, 123501 (2011)

16. S. Carneiro, H.A. Borges, JCAP 1406, 010 (2014)

17. K. Koyama, R. Maartens, Y.S. Song, JCAP 0910, 017 (2009)

18. D. Wands, J. De-Santiago, Y. Wang, Class. Quantum Gravity 29. 145017 (2012)

19. W. Zimdahl, H.A. Borges, S. Carneiro, J.C. Fabris, W.S. HipolitoRicaldi, JCAP 1104, 028 (2011)

20. M. Kunz, Phys. Rev. D 80, 123001 (2009)

21. I. Wasserman, Phys. Rev. D 66, 123511 (2002)

22. C. Rubano, P. Scudellaro, Gen. Relat. Gravit. 34, 1931 (2002)

23. G. 't Hooft, Phys. Rep. 142, 357 (1986)

24. W. Zimdahl, D.J. Schwarz, A.B. Balakin, D. Pavón, Phys. Rev. D 64, 063501 (2001)

25. R.R.R. Reis, I. Waga, M.O. Calvão, S.E. Jorás, Phys. Rev. D 68 , 061302 (2003)

26. S. del Campo, R. Herrera, D. Pavón, JCAP 0901, 020 (2009)

27. I.L. Shapiro, J. Solà, Phys. Lett. B 682, 105 (2009)

28. L.P. Chimento, Phys. Rev. D 81, 043525 (2010)

29. M.L. Tong, H. Noh, Eur. Phys. J. C 71, 1586 (2011)

30. F. Arevalo, A.P.R. Bacalhau, W. Zimdahl, Class. Quantum Gravity 29, 235001 (2012)

31. N. Komatsu, S. Kimura, Phys. Rev. D 89, 123501 (2014)

32. V. Salvatelli, N. Said, M. Bruni, A. Melchiorri, D. Wands, Phys. Rev. Lett. 113, 181301 (2014)

33. D.G.A. Duniya, D. Bertacca, R. Maartens, Phys. Rev. D 91, 063530 (2015)

34. R.S. Gonçalves, G.C. Carvalho, J.S. Alcaniz, Phys. Rev. D 92, 123504 (2015)

35. R. Murgia, S. Gariazzo, N. Fornengo, JCAP 1604, 014 (2016)

36. H.A. Borges, S. Carneiro, J.C. Fabris, W. Zimdahl, Phys. Lett. B 727, 37 (2013)

37. S. Carneiro, C. Pigozzo, JCAP 1410, 060 (2014)

38. C. Pigozzo, S. Carneiro, J.S. Alcaniz, H.A. Borges, J.C. Fabris, JCAP 1605, 022 (2016)

39. I. Dymnikova, M. Khlopov, Eur. Phys. J. C 20, 139 (2001)

40. R.J. Scherrer, Phys. Rev. Lett. 93, 011301 (2004)

41. A.J. Christopherson, K.A. Malik, Phys. Lett. B 675, 159 (2009)

42. J.S. Alcaniz, H.A. Borges, S. Carneiro, J.C. Fabris, C. Pigozzo, W. Zimdahl, Phys. Lett. B 716, 165 (2012)

43. N.C. Devi, H.A. Borges, S. Carneiro, J.S. Alcaniz, MNRAS 448, $37(2015)$

44. H. Velten, H.A. Borges, S. Carneiro, R. Fazolo, S. Gomes, MNRAS 452, 2220 (2015)

45. T.A. Ferreira, C. Pigozzo, S. Carneiro, J.S. Alcaniz, arXiv:1712.05428 [astro-ph.CO] 
46. Z. Zhai, M. Blanton, A. Slosar, J. Tinker, Astrophys. J. 850, 183 (2017)

47. U. Alam, V. Sahni, A.A. Starobinsky, JCAP 0304, 002 (2003)

48. S. Carneiro, H.A. Borges, Gen. Relat. Gravit. 50, 1 (2018)

49. J.A. Frieman, C.T. Hill, A. Stebbins, I. Waga, Phys. Rev. Lett. 75, 2077 (1995)

50. V. Sahni, S. Habib, Phys. Rev. Lett. 81, 1766 (1998)

51. Y.B. Zeldovich, JETP Lett. 6, 316 (1967)

52. G. 't Hooft, arXiv: gr-qc/9310026

53. L. Susskind, J. Math. Phys. 36, 6377 (1995)

54. G. Veneziano, Phys. Lett. B 454, 22 (1999)

55. R. Tavakol, G. Ellis, Phys. Lett. B 469, 37 (1999)

56. R. Easther, D. Lowe, Phys. Rev. Lett. 82, 4967 (1999)

57. N. Kaloper, A. Linde, Phys. Rev. D 60, 103509 (1999)

58. A.G. Cohen, D.B. Kaplan, A.E. Nelson, Phys. Rev. Lett. 82, 4971 (1999)

59. R. Bousso, Class. Quantum Gravity 17, 997 (2000)

60. R. Bousso, JHEP 0011, 038 (2000)
61. D. Bak, S.J. Rey, Class. Quantum Gravity 17, L83 (2000)

62. G.A. Mena Marugán, S. Carneiro, Phys. Rev. D 65, 087303 (2002)

63. G.W. Gibbons, S.W. Hawking, Phys. Rev. D 15, 2738 (1977)

64. M. Spradlin, A. Strominger, A. Volovich, arXiv:hep-th/0110007

65. T. Padmanabhan, Class. Quantum Gravity 21, 4485 (2004)

66. A.Y. Kamenshchik, U. Moschella, V. Pasquier, Phys. Lett. B 511, $265(2001)$

67. J.C. Fabris, S.V.B. Goncalves, P.E. de Souza, Gen. Relat. Gravit. 34, 53 (2002)

68. N. Bilic, G.B. Tupper, R.D. Viollier, Phys. Lett. B 535, 17 (2002)

69. M.C. Bento, O. Bertolami, A.A. Sen, Phys. Rev. D 66, 043507 (2002)

70. J.S. Alcaniz, D. Jain, A. Dev, Phys. Rev. D 67, 043514 (2003)

71. S. Carneiro, arXiv: 1801.10548 [gr-qc] 